\title{
Gastrodin improves learning behavior in a rat model of Alzheimer's disease induced by intra- hippocampal Aß 1-40 injection
}

\author{
Xing Liu, Mengya Wang*
}

From 2011 International Conference on Molecular Neurodegeneration

Shanghai, China. 22-24 September 2011

\section{Background}

Gastrodin extracted from the rhizome of Gastrodia elata Blume, a Chinese herbal medicine, has long been used for treating vertigo, general paralysis, epilepsy, tetanus, stroke and dementia. Although several reports have shown that gastrodin has neuroprotective effects to rescue leadinduced synaptic plasticity deficits in rat hippocampus, and hippocampal cell damage in cellular model of Alzheimer's disease induced by A $\beta 25-35$, its effect on behaviors of rat model of Alzheimer's disease induced by intrahippocampal $A \beta$ injection is not studied yet.

\section{Methods}

Forty-one male adult Sprague-Dawley rats were randomly divided into groups: normal $(n=10)$, sham operated $(n=7$, intra-hippocampal saline injection), saline ( $n=8$, intrahippocampal $A \beta$ injection and then ig saline), gastrodin $(n=8$, intra-hippocampal $\mathrm{A} \beta$ injection and then ig gastrodin), huperzine $\mathrm{A}(n=8$, intra-hippocampal $\mathrm{A} \beta$ injection and then ig huperzine $A)$. One week after intra-hippocampal A $\beta 1-40$ injection ( $5 \mu \mathrm{g}$ in $1 \mu \mathrm{l} \mathrm{PBS}$, bilaterally), gastrodin $(200 \mathrm{mg} / \mathrm{kg})$, huperzine A $(300 \mu \mathrm{g} / \mathrm{kg})$ or saline were administrated by ig for 27 days (q.d.). At end of gastrodin or huperzine A treatment, the 5-day Morris water maze test was performed to observe the learning and memory function of 5 groups of animals.

\section{Results}

Two-way ANOVA (repeated measures) was used to compare the difference of escape latency in place navigation trials among testing days or groups, and showed both differences among 5 test days and 5 groups were very significant $(P<0.001)$. Bonferroni posttests indicated that the difference between normal and sham operated was not significant $(P>0.05)$, but the latency in saline group was significantly longer than normal or sham operated groups $(P<0.05$ or $P<0.01)$, suggesting establishment of rat model. Compared to other groups, the latency in gastrodin group at $2^{\text {nd }}$ test day was significantly shorter than saline group $(P<0.05)$, indicating learning improvement of rat model. However, the latency in huperzine A group was observed to be longer than normal group $(P<0.01)$. In spatial probe trial after 5 -day place navigation trials, difference of numbers to cross the assumed platform among 5 groups was not significant (One-way ANOVA, $P>0.05$ ).

\section{Conclusion}

Gastrodin may have therapeutic effect to improve learning behavior in rat model of Alzheimer's disease induced by intra-hippocampal $A \beta 1-40$ injection by mechanism different from huperzine $\mathrm{A}$.

\section{Acknowledgements}

This work was supported by the NSF of Anhui Province, China (090413084).

Published: 7 February 2012

\section{doi:10.1186/1750-1326-7-S1-S15}

Cite this article as: Liu and Wang: Gastrodin improves learning behavior in a rat model of Alzheimer's disease induced by intra-hippocampal $A \beta$ 1-40 injection. Molecular Neurodegeneration 2012 7(Suppl 1):S15.

\footnotetext{
* Correspondence: wangmy@wnmc.edu.cn

Cell Electrophysiology Laboratory, Wannan Medical College, Wuhu, Anhui 241002, China
} 